\title{
The Hispanic and Luso-Brazilian World: El Proceso de Convertirse en Hombre en las Historias de Piri Thomas y Pedro Juan Soto.
}

Antonio Medina-Rivera

Cleveland State University, a.medinarivera@csuohio.edu

Follow this and additional works at: https://engagedscholarship.csuohio.edu/clmlang_facpub

Part of the Latin American Literature Commons

How does access to this work benefit you? Let us know!

Publisher's Statement

Hispania (C) 2003 American Association of Teachers of Spanish and Portuguese

\section{Recommended Citation}

Medina-Rivera, Antonio. "The Hispanic and Luso-Brazilian World: El Proceso De Convertirse En Hombre En Las Historias De Piri Thomas Y Pedro Juan Soto." Hispania. 86.4 (2003): 898-906.

This Article is brought to you for free and open access by the Department of World Languages, Literatures, and Cultures at EngagedScholarship@CSU. It has been accepted for inclusion in World Languages, Literatures, and Cultures Faculty Publications by an authorized administrator of EngagedScholarship@CSU. For more information, please contact library.es@csuohio.edu. 


\title{
THE HISPANIC AND LUSO-BRAZILIAN WORLD EL PROCESO DE CONVERTIRSE EN HOMBRE EN LAS HISTORIAS DE PIRI THOMAS Y PEDRO JUAN SOTO
}

\author{
Antonio Medina-Rivera, Cleveland State University
}

This article was originally published in:

Medina-Rivera, Antonio (2003). The Hispanic and Luso-Brazilian World El Proceso de Convertirse en Hombre en las Historias de Piri Thomas y Pedro Juan Soto. Hispania, 86(4), 898-906.

Post-print standardized by MSL Academic Endeavors, the imprint of the Michael Schwartz Library at Cleveland State University, 2013

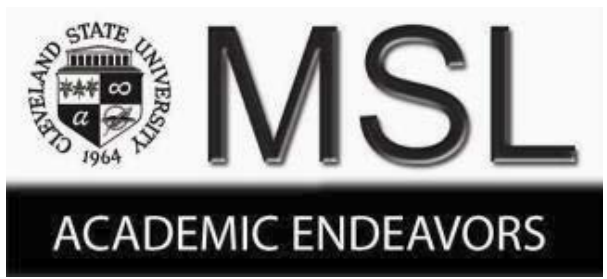




\title{
THE HISPANIC AND LUSO-BRAZILIAN WORLD
}

\section{El proceso de convertirse en hombre en las historias de Piri Thomas y Pedro Juan Soto ${ }^{1}$}

\author{
Antonio Medina-Rivera \\ Cleveland State University
}

\begin{abstract}
En este estudio se examina el proceso de convertirse en hombre en el cuento "Campeones" de Pedro Juan Soto y en la autobiografia Down These Mean Streets de Piri Thomas. Varios de los elementos que aparecen en el movimiento masculino sirven como punto de partida para esta investigación: la violencia, la figura del héroe y el distanciamiento entre padre e hijo. En el análisis se ven las figuras masculinas desde la perspectiva del varón que pertenece a un grupo minoritario dentro del conglomerado multicultural de los Estados Unidos. Con este estudio se pretende ofrecer una visión más amplia del varón hispano, la cual pretende ir más allá de la imagen del "macho" latinoamericano y seguir abriendo campo a los estudios masculinos en la literatura hispanoamericana.
\end{abstract}

Key Words: estudios masculinos, héroe, Thomas (Piri), Soto (Pedro Juan), machismo, violencia, guerrero, Bly (Robert), Down These Mean Streets, Spiks

os estudios masculinos de los años noventa plantean como punto de interés el redefinir la imagen del varón en la sociedad moderna. Para esto se valen de arquetipos universales, fábulas, leyendas e historias milenarias que sirven para explicar la conducta del varón. La imagen del héroe o guerrero que popularizó Robert Bly con su libro Iron John durante los primeros años de la década de los noventa parecía ser el arquetipo que representaba de una manera más adecuada la imagen del varón norteamericano. Esta imagen de guerrero/héroe parecía ser una manera adecuada y atractiva para representar al varón dentro de la sociedad moderna. La misma la ha heredado el varón a través de generaciones anteriores, de culturas primitivas, y ha llegado a nosotros a través del inconsciente colectivo. Con respecto a esta imagen, Sam Keen comenta que "the male mind-set is a warrior psyche because society demands that we constantly resort to power and violence" (37).

Como era de esperarse, no faltaron las críticas a la imagen del guerrero. Por una parte feministas, ecologistas y pacifistas criticaron los elementos de violencia y dominación que se asocian a las figuras de este arquetipo. Por otro lado, algunos grupos minoritarios, según comenta Allan B. Chinen en su libro Beyond the Hero, "rise up against the patriarch and dispute his insistence on one law, one culture, and one doctrine for everyone" (1). La imagen del guerrero o del héroe parecía perpetuar la idea de un varón dominante y violento, y parecía ser una manera más de justificar el patriarcalismo, el machismo y la superioridad del hombre frente a la mujer.

Junto a los estudios que toman como punto de partida al héroe o guerrero, se encuentran también los que tratan de explicar algunos de los problemas sicológicos que afectan el desarrollo pleno del varón (e.g., Robert Hicks y una amplia lista de sicólogos y teólogos junguianos). Estos rescatan los elementos positivos que se asocian a la imagen del guerrero (lealtad, perseverancia, valor, etc.), y rechazan aquellos elementos que impiden el crecimiento pleno del varón y que obstaculizan su relación con la mujer y con el resto de la sociedad (dominación, machismo, violencia). Hicks ve la imagen del guerrero como una etapa dentro del proceso de convertirse en hombre, etapa a la cual le suceden otras de mayor crecimiento y madurez en el desarrollo del varón. 
En vez de estudiar la conducta del varón a partir de arquetipos estáticos y que deberían acomodarse a la conducta de todo varón, Hicks hace un acercamiento procesual y usa distintos arquetipos que se asocian a diferentes etapas en el proceso de maduración del varón. Hicks habla de etapas en el proceso de convertirse en hombre, y para cada una de esas etapas presenta un arquetipo diferente:

(1) El hombre-creatura: Este es el salvaje noble que está conectado a la creación, a la idea de haber sido creado a imagen y semejanza de Dios y en igualdad con la mujer. Se relaciona a la etapa en la que Adán convivía con Eva en el Paraíso. Podría también referirse a los primeros años de edad en un varón, en los cuales aún no se ha descubierto la autodiferenciación con respecto a la mujer.

(2) El hombre fálico: Esta es la etapa del descubrimiento y despertar de la sexualidad en la cual el varón se autodefine en relación a su pene. Esta etapa se empieza a percibir desde los primeros años de la adolescencia en las cuales la atención del varón se concentra en los cambios físicos que experimenta, el falo se convierte en su obsesión y centro de su autodefinición como hombre.

(3) El guerrero: Esta etapa coincide hasta cierto punto con la anterior y hasta podría antecederla. El varón crea conciencia de su fuerza, y de su deseo de competir y ganar. Se percibe no sólo en el interés por el deporte, sino que también en su afán de competencia en el trabajo y en casi todos los aspectos de la vida.

(4) El hombre herido: Esta etapa se experimenta durante los primeros años de adultez del varón cuando éste, o bien no ha podido alcanzar las metas que se planteó, o se ha tenido que enfrentar a las dificultades mayores que se dan en la adultez y en la ubicación permanente en la vida, o como producto del conflicto intergeneracional.

(5) EI hombre maduro: A esta etapa llega el varón que supo enfrentarse a los conflictos de la etapa anterior, supo manejar las heridas del pasado, e integró todas las dificultades a su proceso de crecimiento y madurez.

(6) El hombre sabio: Esta es la etapa óptima en el desarrollo de un varón y no sólo implica la integración y equilibrio de todas las etapas anteriores, sino que también el sabio toma el rol de mentor, consejero y guía de otros varones que están desarrollándose.

De acuerdo a Hicks, el sabio sería la etapa de plenitud en el desarrollo del varón, pero muchos se quedan estancados en la etapa fálica, en la del héroe o en la del hombre herido. Aunque el enfoque de Hicks es totalmente espiritual, la caracterización del varón como una serie de etapas ayuda a visualizar al varón de una manera más objetiva y multidimensional. De esta manera no se limita la imagen del varón a un solo arquetipo o estereotipo que nos ofrece una visión limitante y de carácter homogéneo. Otros investigadores, al igual que Hicks, han querido ir más allá de la imagen del héroe o guerrero, y añaden otros arquetipos como el sabio, el sacerdote o el curandero para no excluir al varón que no se identifica con el arquetipo del guerrero (e.g., Chinen).

El principal problema del varón en la sociedad moderna, de acuerdo a muchos de los escritores del movimiento masculino, es el distanciamiento que existe entre el padre y el hijo, y la incapacidad del varón para subsanar este distanciamiento. Partiendo de este distanciamiento, tanto Hicks como otros investigadores de su generación, apuntan de una manera directa o indirecta al proceso de convertirse en varón en distintas culturas del mundo.

Las historias de los escritores hispanos Piri Thomas y Pedro Juan Soto relatan el doloroso, interesante, jocoso y traumatizante proceso de convertirse en varón. Ambos hablan desde la perspectiva del grupo minoritario, en este caso, el puertorriqueño que vive en Nueva York. En el siguiente trabajo se examinará la manera en que las historias de estos dos escritores reflejan las ideas básicas que exponen los escritores del movimiento masculino, a partir de la perspectiva y matices del que vive y pertenece a un grupo minoritario. El análisis se limitará al cuento "Campeones" de Pedro Juan Soto de su colección de cuentos Spiks y a la autobiografía Down These Mean Streets de Piri Thomas. Ambas historias relatan el proceso de convertirse en hombre en una sociedad conflictiva como lo es el barrio latino de la ciudad de Nueva York. 
Pedro Juan Soto nació en Puerto Rico. Su inclinación universitaria lo llevó a Nueva York a estudiar prémedica, carrera que abandonó por su pasión por las letras. En las décadas de los cincuenta y los sesenta publicó una serie de libros en los que recoge el choque sicológico y cultural que se da en el proceso de aculturación de los puertorriqueños en Nueva York. Según Luz M. Umpierre, en su entrevista con Pedro Juan Soto, él mismo señala que "la literatura, desde su inicio, tiene la función de la denuncia, de la impugnación de la sociedad" (591), y es precisamente esto lo que vemos en las historias de Soto. Su colección de cuentos Spiks, quizás su libro más reconocido, se publicó en 1957 y en el mismo se recoge de una manera cinematográfica la dura realidad del puertorriqueño que vive en Nueva York.

Posterior a Soto, aparece la figura de Piri Thomas quien alcanzara fama en los Estados Unidos a partir del 1967 con la publicación de su obra clásica Down These Mean Streets. En la misma, relata sus primeros años de vida y sus años en una cárcel tras haber herido a un policía de Nueva York. En palabras de Rodríguez de Laguna, "Down These Mean Streets is the tale of a mulatto Puerto Rican youth in search of his racial definition and cultural identity who wanders uncomfortably in the contested social, cultural, and racial spaces at home, the school, and the 'mean' streets" (22). El tema de la búsqueda de la identidad y la autoaceptación también están presentes en otros cuentos de Piri Thomas tal como lo señala Eugene V. Mohr: "Underlying and uniting the data is the powerful theme that runs through all of Thomas's work: the painful job of recognizing and accepting oneself in a world of confusing norms and tangled ideologies" (62).

Tanto Soto como Thomas representan la vida del puertorriqueño que vive en Nueva York, aunque partiendo de experiencias y perspectivas diferentes. Soto escribe como testigo ocular de la realidad. Su perspectiva es la del observador que percibe la realidad desde una distancia. Representa estampas de la vida neoyorkina, pero al estilo del neorrealismo italiano de la época, se limita a describir lo que ve, y hasta cierto punto denunciar, sin recurrir al comentario moralista, político o social. Los cuentos de Spiks dejan al lector con una imagen fotográfica. Hay más detalles visuales de lo que el autor logra relatar en su narración, y corresponde al lector interpretar y observar cuidadosamente la imagen para llegar a sus conclusiones, o en ocasiones se ve obligado a completar la parte de la historia que es parte de la imagen, pero que no ha sido narrada por el autor. Thomas, por su lado, escribe no como testigo, sino como participante de la realidad neoyorkina. Lo que sufre la comunidad puertorriqueña, Thomas lo ha experimentado en carne propia. Su prosa es menos técnica y trabajada que la de Soto, aunque con una variedad única de registros (Mohr 73); sin embargo, la riqueza de las imágenes sensoriales y la honestidad de su prosa llevan al lector a identificarse plenamente con él.

Este trabajo no se limita al tema de la identidad cultural que tanto caracteriza a los críticos que analizan las obras de Soto y Thomas (e.g., Umpierre, Sandoval y Seda Bonilla en el caso de Pedro Juan Soto; Mohr y Rodríguez en el caso de Thomas) aunque lo utilice como punto de partida para investigar la formación del constructo de género masculino en el contexto del choque cultural. Como mismo señala Umpierre, "las obras de Pedro Juan Soto, y podríamos decir que las de muchos escritores puertorriqueños, se prestan a nuevas interpretaciones no tradicionales dentro del proceso crítico isleño" ("El francotirador" 7). En este ensayo, se pondrá énfasis en los personajes masculinos que tanto sobresalen en las obras de Soto y Thomas. En ambos autores, el carácter y la angustia del varón se trabajan cuidadosamente. Ninguno representa al varón dominante, poderoso y controlador; sino al varón subyugado por el sistema y por la cultura dominante de los Estados Unidos, el varón sumergido en la desesperación, pasivo en muchas ocasiones y violento en otras, derrotado e incapacitado de sobresalir y acomodarse dentro de la sociedad estadounidense. A estos varones, costaría percibirlos desde la perspectiva del arquetipo del héroe o el guerrero que nos presentan los estudios masculinos de los Estados Unidos. Son varones sometidos, sin poder político, representantes de grupos minoritarios en los Estados Unidos, y que descubren el significado de ser y convertirse en hombre mediante las experiencias duras y crudas a las que tienen que enfrentarse para sobrevivir día a día. 
El proceso de convertirse en hombre está marcado en muchas sociedades primitivas por ritos de iniciación que le marcan al varón ese proceso. Varios escritores del movimiento masculino, entre los que podemos destacar a Keen, señalan que la falta de ritos de iniciación entre los varones de sociedades modernas impide que estos puedan determinar con exactitud las expectativas que tiene la sociedad sobre ellos y el definir con claridad lo que significa e implica ser hombre. Los personajes de Soto y Thomas servirán para analizar cómo se da este proceso de convertirse en varón en una sociedad conflictiva como Nueva York y bajo el estatus de minoría, que caracteriza al puertorriqueño que sobrevive día a día en ese ambiente.

\section{El cuento "Campeones" de Pedro Juan Soto}

En el cuento "Campeones," Soto presenta a dos jugadores de billar, Gavilán y Puruco. Gavilán es un hombre maduro quien acaba de salir de la cárcel luego de un período de seis meses, y el cual es reconocido en el barrio como el campeón en el deporte de los tacos. Puruco tiene sólo 16 años y es la nueva sensación del billar, y le acaba de ganar dos mesas a Gavilán. En las propias palabras del narrador, "Era el nuevo, el sucesor de Gavilán y los demás individuos respetables. Era igual... No. Superior, por su juventud: tenía tiempo y oportunidades para sobrepasar todas las hazañas de ellos" (72). Gavilán, en vez de asumir su misión como mentor o sabio, de acuerdo a las etapas del varón que presenta Hicks, se siente amenazado y desplazado por la habilidad de Puruco.

En el barrio, ser campeón de billar es signo de prestigio y respeto, y sirve de trampolín para otros negocios y conexiones: "De ahí, a la fama: el macho del barrio, el individuo indispensable para cualquier asunto - la bolita, el tráfico de narcóticos, la hembra de Riverside Drive de paseo por el barrio, la pelea de esta pandilla con la otra para resolver "cosas de hombres"' (74). Al enfrentarse por tercera vez, Puruco siente que Gavilán está recurriendo a la brujería para poder aventajarlo y finalmente se da cuenta que éste recurre a "jugar sucio" para ganarle. Cuando Puruco reclama, Gavilán lo humilla para mantener su prestigio de macho campeón: "¿Tú te creeh que un pilemielda como tú me va a llamar a mí tramposo? - dijo Gavilán, forzando sobre el pecho de Puruco el puño que desgarraba la camiseta - Te dejo ganar doh mesitah pa que tengas de qué echártelah, y ya te creeh rey. Echa p'allá, infelih - dijo entre dientes- . Cuando crehcas noh vemo" (79).

Para Puruco, el proceso de convertirse en hombre implica competencia y violencia. El ser un buen jugador de billar lo llevará a dar un paso gigante en ese proceso, pero eso no basta, pues tiene que enfrentarse al orgullo herido de los veteranos, a los cuales se siente sometido por diferencia de edad, poderío físico y conexiones ilícitas en el barrio. Desde el punto de vista de los ritos de iniciación, no encuentra quien lo inicie, no hay un padre que lo guíe, y el mentor o sabio correspondiente lo ve como competencia. En este sentido, Puruco tiene que iniciarse por sí solo a la vida.

Al final de la historia, Puruco se enfrenta a un niño menor que él, que se interpone en su camino para recoger el aro de un barril. Puruco corresponde dándole un manotazo al niño y diciéndole, "Cuidao, nen, que te parto un ojo" (80). Puruco, al igual que Gavilán, muestra su supremacía de una manera violenta. La ley que impera en el barrio es la misma del darwinismo en la que sólo el más fuerte sobrevive. Ser hombre implica mostrar fuerza y poderío físico, en un ciclo en en cual el más fuerte siempre encuentra a uno más débil para humillarlo y someterlo a su voluntad.

\section{La autobiografía Down These Mean Streets de Piri Thomas}

En Down These Mean Streets también se percibe el proceso de un niño que se convierte en hombre. Al principio de la historia ya vemos como Piri es un niño de la calle, que sobrevive y busca el respeto de los otros varones del barrio. Piri recurre aún más a la calle cuando su padre se ve incapacitado de servirle como mentor. Este se limita a servir de proveedor, pero como muchos otros padres no considera la posibilidad de iniciar a sus hijos varones a la vida. Piri recurre a las 
pandillas y eventualmente a la vida criminal. Dentro de su proceso de crecimiento, Piri se plantea su identidad racial, pues al ser un joven mulato, lo confunden en muchas ocasiones con una persona de raza negra. Piri vuelve al padre para recibir apoyo en su proceso de aceptación y reafirmación racial, pues su padre también es mulato, pero el padre se siente ajeno ante los reclamos de Piri y le dice: "Maybe you see something in me I haven't seen yet, or maybe won't admit yet. I don't like feeling to be a black man. Can you understand it's pride to me being a Puerto Rican?" (150). Con la respuesta del padre se crea el distanciamiento y la falta de entendimiento entre los dos. Para convertirse en hombre, Piri tendrá que seguir recurriendo a la calle. A esto comenta Rodríguez de Laguna, "It is precisely in the home - the family space par excellence at the core of Hispanic culture and values - where Piri ironically initially encounters loneliness, rejection, and invisibility. He is the darkest of all his siblings, and because of his color he is the target of his black father's cold treatment and resentment" (23).

Luego de este incidente, Piri cuenta con el apoyo del sabio o mentor, representado en la figura de su amigo afroamericano de nombre Brewster Johnson quien, aunque reconoce la herencia negra en Piri, lo trata de convencer de que se reconozca como joven hispano, pues él mismo ha sufrido en sangre propia la desventaja de ser negro en una sociedad marcada por el racismo. Ante la insistencia de Piri, Brewster lo acompaña en un viaje al sur de los Estados Unidos, en un proceso que lo ayudará a descubrir la realidad del negro estadounidense, realidad que Brewster conoce muy bien por haberse criado en el sur. Cuando desaparece Brewster, a raíz de un incidente inexplicable, Piri se queda solo para enfrentarse al conflictivo mundo que lo rodea, hasta que finalmente termina en la prisión por atentar contra la vida de un policía.

En su proceso para convertirse en hombre Piri experimenta distanciamiento con el padre, diferenciación y falta de aceptación social por su color y por pertencer a un grupo minoritario. Se enfrenta también al pandillerismo, a la promiscuidad, a la vida criminal, pero sin pasar por un rito de iniciación que lo lleve a disfrutar de su "ser hombre" a plenitud.

\section{Discusión:}

En Soto y en Thomas, a pesar de las diferencias en la perspectiva, se dan muchas similaridades al interpretar el proceso de convertirse en hombre. En ambos se da el tema de la violencia, se desprende hasta cierto punto la imagen del héroe y se da la ausencia o distanciamiento del padre.

Violencia y agresividad: La violencia forma una parte esencial en el proceso de convertirse en hombre, pues es la manera principal de mantener reputación y prestigio como macho en el barrio. Tanto Puruco como Piri caen dentro de ese ciclo de violencia en el cual primero fueron víctimas y luego agresores. Puruco sufre la violencia de Gavilán, y al final del cuento agrede a un niño que se le mete en el medio del camino. Inclusive, está consciente de la violencia en su vida: "Era un hombre ya. Podía hacer daño, mucho daño, y también podía sobrevivir a él" (79).

Piri desde niño experimenta la violencia en el barrio, desde sus conflictos con los niños italianos que le servían de antagonistas a los niños hispanos durante esa época, hasta el uso de la violencia para ser aceptado en una pandilla de niños hispanos. La violencia entre sus propios compañeros le sirve para ganar día a día respeto y aceptación.

Las imágenes de guerrero, hombre salvaje o cazador con las que se suele identificar al hombre de épocas milenarias están directamente relacionadas al uso de la violencia y de la fuerza. La violencia se emplea para defender el orgullo propio y para defender el honor. Chinen sugiere que "Men become violent out of desperation, when their hidden humiliation is exposed" (39). Gavilán se vuelve violento al ver amenzado su orgullo de campeón. Puruco se violenta a su vez tras experimentar la humillación por parte de Gavilán y para mostrar a la vez su supremacía frente al más pequeño. Para Piri, la violencia es una necesidad, que como dice Chinen surge como consecuencia de la desesperación. La violencia, en su caso, es un elemento de supervivencia y de permanencia dentro del barrio.

Junto a la violencia es necesario hablar de la agresividad, la cual parece acompañar al varón en su desarrollo. Se ve en la práctica de deportes como el fútbol americano o en la formación que 
recibe un militar. Hicks describe la agresividad como elemento esencial en la etapa del hombre guerrero. Esta etapa se suaviza, según Chinen, en la medida en la que el varón vaya descubriendo su lado femenino después de la adolescencia. Hay que señalar, sin embargo, que la agresividad no siempre conduce a la violencia. La agresividad se puede ver como rasgo de la personalidad de un individuo, mientras que la violencia se percibiría como una deficiencia o desajuste de tipo sicológico.

La agresividad como rasgo del carácter masculino tiene su lugar en el desarrollo del varón. Bly sugiere que en tiempos milenarios esta agresividad se encausaba mediante las actividades típicamente masculinas como la caza y la guerra. En la actualidad esa energía que el varón ha heredado se debería liberar a través del deporte y de otras actividades físicas, que según Bly contribuyen al desarrollo pleno del varón. Gavilán, Puruco y Piri manifiestan su agresividad en su interacción con otros varones del barrio, agresividad que en estos casos específicos toma la forma de violencia.

Bly, Hicks y Chinen al hablar del guerrero, de la violencia y de la agresividad, no toman en consideración las condiciones socioeconómicas, discriminación y carencia de oportunidades que suelen sufrir los varones de grupos minoritarios en sociedades como la realidad que se vive en los Estados Unidos. Tampoco se puede descartar el impacto de los medios de comunicación social y la cultura de violencia que estos han generado en los últimos años. Los personajes de Thomas y Soto corresponden al estrato más bajo dentro de la sociedad, en donde la violencia se convierte en un estilo de vida y de supervivencia.

Tampoco hay que generalizar que esta conducta violenta es característica del machismo latinoamericano. Primero, porque como comenta Rafael Ramírez en su estudio sobre el varón puertorriqueño: "Not all men are violent, agressive, and oppressive" (113), y segundo, porque como dice Martín Espada: "While Latino male behavior is, indeed, all too often sexist and violent, Latino males in this country are in fact no worse in that regard than their Anglo counterparts. Arguably, European and European-American males have set the world standard for violence in the twentieth century, from the Holocaust to Hiroshima to Vietnam" (88).

El héroe y la búsqueda de reconocimiento: De la imagen del guerrero se desprende a su vez la imagen del héroe. En las historias de Soto y Thomas se podría afirmar que el concepto del héroe está latente. En el caso de Puruco, el convertirse en campeón de billar, lo llevará a ser reconocido como héroe. Como señala Soto en su introducción al cuento "Puruco es el adolescente que aspira a conquistar 'el mundo' pese a las múltiples humillaciones que pueda sufrir en su empeño" (71). Su condición de héroe, sin embargo, por las condiciones sociales y conflictivas en las que se dan, se vería siempre amenazada por el desplazamiento de otros nuevos jugadores con más destreza y habilidad. Para Piri, la imagen del héroe no se dará sino hasta que llegue a la cárcel, se descubra a sí mismo y vea la necesidad de recibir reconocimiento como él mismo dice en el prólogo de su libro "I wanna tell ya I'm here-you bunch of mother-jumpers - I'm here, and I want recognition, whatever that mudder-fuckin word means" (ix).

El alcanzar estatus de héroe está ligado directamente al proceso de convertirse en hombre. Dentro de la cultura dominante, partiendo de una visión neoliberalista, la figura del héroe se relaciona al éxito individual, financiero y adquisitivo, como señala Warren Steinberg: "High achievement and ambition to be successful in one's work are hallmarks of the masculine gender role. Men are expected to be successful, seek higher status, and be looked up to. American men must achieve wealth, fame, or recognition" (46). En el caso de Puruco y Piri, lo que importa es el ser reconocido dentro de una sociedad que margina, discrimina y no les brinda las oportunidades necesarias. El reconocimiento y la aceptación, antes que el éxito financiero o en adición a éste, parecen ser ingredientes necesarios en la imagen del héroe para el varón que pertenece a un grupo minoritario.

En Down These Mean Streets desfilan las imágenes de los antihéroes que jamás serán reconocidos. Tanto el padre de Piri como su amigo Brewster se estancan en la etapa del héroe herido de la que nos habla Hicks. El padre de Piri se acomoda al conformismo y a su incapacidad de verse a sí mismo tal cual él es, y por ende carece de la capacidad de conducir a sus hijos varones por el 
camino de la autoaceptación. Brewster carga con historias de dolor y discriminación, en una sociedad anterior al desarrollo de los derechos civiles de los negros en los Estados Unidos. Para convertirse en héroes, tanto el padre de Piri como Brewster tendrían que enfrentarse al sistema opresivo que les ha tocado vivir.

Gavilán y Puruco experimentan por su lado el sabor de sentirse héroes, o al menos ante los ojos de la realidad del barrio lo son. Sin embargo, su heroísmo está marcado por un círculo de violencia del cual son tanto víctimas como agresores. Este heroísmo no es reconocido fuera de los límites del barrio, ni siquiera por todos los individuos que viven en el barrio. También Puruco y Gavilán tienen las características del héroe herido, y al no sobrellevar las heridas típicas de esa etapa eventualmente se verán desplazados por los nuevos héroes/antihéroes que vayan surgiendo dentro del conflictivo mundo del billar que se juega en el barrio.

La ausencia del padre: La ausencia del padre o el distanciamiento entre padre e hijo, tema central del movimiento masculino de los Estados Unidos, está también presente en las historias de Soto y Thomas. Este distanciamiento y la carencia de un mentor que ocupe dicho lugar, nos conecta con la imagen del hombre herido del que nos habla Hicks: "It is as if for several generations men have not had fathers and mentors in their lives to teach them how to handle woundedness" (99). Las heridas que marcan el devenir sicológico del varón tienen, hasta cierto punto, su origen en ese distanciamiento de la figura paterna.

En el cuento "Campeones," Puruco, al no tener con quien sanar sus heridas, se desquita con el chiquillo que se encuentra en la calle. Soto no hace mención al padre de Puruco, más bien lo presenta como un niño de la calle, sin dirección y con la única ambición de alcanzar el reconocimiento y la fama que le darán prestigio en el barrio. Generalmente, Soto no indaga en la vida de sus personajes; los presenta como siluetas o estampas de una realidad cruda y dura, de la que él sólo es testigo ocular. Sin embargo, "la ausencia de un padre" se da en la manera en la que se conduce el personaje. El deseo de Puruco de alcanzar fama y reconocimiento fuera del hogar, y a tan temprana edad, no son más que un signo de ese distanciamiento.

Piri, por su parte, recurre a su padre al enfrentarse a sus conflictos de identidad racial, pero éste no sabe corresponder a las inquietudes y angustias del hijo. Brewster se convierte en mentor de Piri, pero al desaparecer, Piri se queda solo y herido, y al igual que Puruco, se desahoga a través de la violencia. Para Hicks el hombre herido es simplemente una etapa dentro del desarrollo del varón, que generalmente se relaciona a la relación padre-hijo, y que al superarse lo conduce a la etapa de madurez y luego a la de sabio o mentor. Gavilán, al no aceptar el crecimiento de Pururo, deja relucir sus complejos, muestra síntomas de hombre herido, y por ende no puede asumir el papel de mentor. En el caso del padre de Piri, los síntomas de hombre herido se dan en el campo laboral, tras la pérdida del trabajo, y en su incapacidad de aceptar su negritud. Eso le impide servirle de mentor a su propio hijo.

Gordon Dalbey señala que la ruptura que existe entre padre e hijo es uno de los impedimentos básicos en la jornada de un hombre para convertirse en varón y comenta que "Clearly, in the most crucial occasion of father-son definition - when the son becomes a father himself-the American man is abandoned. He thus feels inadequate and afraid as a father himself, and therefore, all tooready to abandon his own son" (ix). Este distanciamiento se debe a una especie de rivalidad que se desarrolla entre padre e hijo, rivalidad que lleva a la competencia y finalmente a la incapacidad de comunicarse.

El hombre herido del que nos habla Hicks no es sino el resultado de ese distanciamiento, y a su vez Dalbey lo reitera cuando sugiere que "That wound is caused by an epidemic alienation from the father, who is every man's masculine root in this world" (5). Cuando desaparece esa raíz de la que nos habla Dalbey, entonces se le quebranta al varón su proceso de convertirse en hombre, y se refugia en la violencia, el machismo, la crueldad. Parece ser que todo varón en su proceso de convertirse en hombre ha sido herido por otro varón mayor que él, así suele pasar en muchas culturas primitivas, pero con la única diferencia que luego viene una etapa de acompañamiento y mentoría que ayuda al varón a levantarse y a sanar sus heridas. Piri y Puruco, al igual que otros de los hombres heridos de las historias a las que se hace referencia en este trabajo, carecen de un 
proceso de acompañamiento que los ayude y guíe en su desarrollo como hombres.

\section{Conclusión:}

Las historias de Soto y Thomas, muy poco explotadas en la investigación literaria, nos ayudan en la interpretación sociológica del varón latino que vive en una situación de dominación o marginalización, como es el caso de los hispanos que viven en los Estados Unidos. Las ideas, aportaciones y fundamentos teóricos del movimiento masculino de los Estados Unidos son una herramienta útil para lograr esta interpretación; matizando, por supuesto, de acuerdo a la realidad diferente que vive el varón hispano. El arquetipo del héroe es un arquetipo universal que se reinterpreta de acuerdo a los valores y realidades de cada cultura, y que a la vez se relaciona con el proceso de convertirse en hombre dentro de la sociedad. En este caso, me limité a la imagen del héroe y del varón herido, pero al igual que Chinen, coincido en la posibilidad de experimentar con otros arquetipos masculinos que se adapten a otros varones que no se identifican con la imagen del héroe o guerrero.

La violencia y la agresividad, el heroísmo y la búsqueda de reconocimiento, y la ausencia del padre son ingredientes que se hacen presentes en los personajes de Thomas y Soto. Estos ingredientes definen el proceso de convertirse en hombre para un joven que vive en situaciones de desventaja económica y social en los Estados Unidos. Las obras literarias de Thomas y Soto son un reflejo de esa realidad dura a la que se enfrenta el varón en la sociedad.

\section{NOTA}

'La investigación de este trabajo se logró gracias a una beca de viaje que recibí de parte del Centro de Estudios Latinoamericanos de la Universidad de Pittsburgh durante el verano de 2001.

\section{OBRAS CITADAS}

Bly, Robert. Iron John: A Book About Men. Reading, MA: Addison-Wesley, 1990.

Chang-Rodríguez, Eugenio. Latinoamérica: su civilización y su cultura. $2^{\text {nd }}$ ed. New York: Harper Collins, 1991.

Chinen, Allan B. Beyond the Hero. New York: Putnam Books, 1993.

Cornwall, Andrea and N. Lindisfare, Eds. Dislocating Masculinities. London: Routledge, 1994.

Dalbey, Gordon. Father and Son: The Wound, The Healing, The Call to Manhood. Nashville: Thomas Nelson Publishers.

Dumezil, Georges. L'ideologie tripartie des Indo-Europeens. Bruxelles (Berchem): Latomus, 1958.

Espada, Martín. "The Puerto Rican Dummy and the Merciful Son." Muy macho: Latino Men Confront Their Manhood. Ed. Ray González. New York: Anchor Books, 1996. 75-89.

Goldberg, Herb. The New Male: From Self-Destruction to Self-Care. New York: William Morrow and Company, 1979.

Hicks, Robert. The Masculine Journey. Colorado Springs, CO: Navpress, 1993.

Keen, Sam. Fire in the Belly: On Being a Man. New York: Bantam Books, 1991.

Loprete, Carlos A. Iberoamérica: Historia de su Civilización y Cultura. $3^{\text {rd }}$ ed. Upper Saddle River, NJ: Prentice Hall, 1995.

Mirandé, Alfredo. Hombres y Machos: Masculinity and Latino Culture. Boulder, CO: Westview Press, 1997.

Mohr, Eugene V. "Piri Thomas: Author and Persona." Caribbean Studies 20 (1980): 61-74.

Paz, Octavio. "Máscaras Mexicanas." El Laberinto de la Soledad. 13 th ed. México, DF: Fondo de Cultura Económica, 1984. $26-41$.

Ramírez, Rafael. What It Means to Be a Man: Reflections on Puerto Rican Masculinity. New Brunswick, NJ: Rutgers UP, 1999.

Robertson, Robin. C. G. Jung and the Archetypes of the Collective Unconscious. New York: Peter Lang, 1987.

Rodríguez, Joe. "The Sense of Mestizaje in Two Latino Novels." Revista Chicano-Riqueña 12 (1984): $57-63$.

Rodríguez de Laguna, Asela. "Piri Thomas's Down These Mean Streets: Writing as a Nuyorican/Puerto Rican Strategy for Survival." US Latino Literature: A Critical Guide for Students and Teachers. Eds. H. Augenbraum and M. Fernández-Olmos. Wesport, CT: Greenwood Press, 2000. 21-29.

Sandoval, Alberto. “'Mira, que vienen los nuyoricans!: el temos de la otredad en la literatura nacionalista puertorriqueña." Revista de Crítica Literaria Latinoamericana 23 (1997): 307-25.

Seda-Bonilla, Eduardo. “On The Vicissitudes of Being 'Puerto Rican': An Exploration of Pedro Juan Soto's Hot Land, Cold Season.” Revista/Review Interamericana 8 (1978): 116-28.

Soto, Pedro Juan. Spiks. 1956. Río Piedras, PR: n.p., 1973.

Steinberg, Warren. Masculinity: Identity, Conflict, and Transformation. Boston: Shambala, 1993. 
Thomas, Piri. Down These Means Streets. 1967. New York: Vintage, 1997.

Umpierre, Luz M. "Entrevista con Pedro Juan Soto." Hispania 63.3 (1980): 591-92.

-. "El francotirador' de Pedro Juan Soto- la crítica puertorriqueña y la metaficción." Explicación de Textos Literarios 15 (1986-7): 1-7.

Post-print standardized by MSL Academic Endeavors, the imprint of the Michael Schwartz Library at Cleveland State University, 2015 